\title{
An Efficient Content based Image Retrieval: CBIR
}

\author{
Shaziya Khan \\ M. Tech, \\ Computer Science \& Engineering \\ All Saints'College of \\ Engineering Bhopal
}

\author{
Shamaila Khan \\ Department of \\ Computer Science\& Engineering \\ All Saints'College of \\ Engineering Bhopal
}

\begin{abstract}
Due to the exponential growth of image data there is a dire need for innovative tools which can easily manage, retrieve images and images from the large image database. The most common approach which is being used is Content-Based Image Retrieval (CBIR) system. CBIR is the popular image retrieval system by which reterived the targetted image can be retrieved by matching the features of the given image. The goal of this paper is to develop an image retrieval based on content properties such as shape, color, texture etc. usually encoded into feature vectors. One of the main advantages of the CBIR approach is the possibility of an automatic retrieval process instead of the traditional keyword-based approach. The CBIR technology has been used in several applications such as fingerprint identification, biodiversity information systems, digital libraries, medicine and historical research among others. This paper aims to develop a new efficient tool for CBIR based on above mention parameters using MATLAB
\end{abstract}

\section{Keywords}

Image processing, Colour, Size, Shape, Texture, precision and Recall

\section{INTRODUCTION}

Content-based image retrieval (CBIR), also known as query by image content (QBIC) and content-based visual information retrieval (CBVIR) is the application of computer vision techniques to the image retrieval problem, that is, the problem of searching for digital images in large databases. Content-based image retrieval is opposed to traditional concept-based approaches."Content-based" means that the search analyzes the contents of the image rather than the metadata such as keywords, tags, or descriptions associated with the image. The term "content" in this context might refer to colors, shapes, textures, or any other information that can be derived from the image itself. CBIR is desirable because searches that rely purely on metadata are dependent on annotation quality and completeness. Having humans manually annotate images by entering keywords or metadata in a large database can be time consuming and may not capture the keywords desired to describe the image. The evaluation of the effectiveness of keyword image search is subjective and has not been well-defined. In the same regard, CBIR systems have similar challenges in defining success. The internet and digital technologies have imposed a need to have a system to organize abundantly available digital images for easy categorization and retrieval. Databases of art works, satellite and medical imagery have been attracting more and more users in various professional fields for example, geography, medicine, architecture, advertising, design, fashion, and publishing. Effectively and efficiently accessing desired images from large and varied image databases is now a necessity [1].
Fig 1 shows the Architectural view Content based image retrieval system. Retrieval of required-query-similar images from abundantly available accessible digital images is a challenging need of today. The image retrieval techniques based on visual image content has been in-focus for more than a decade. Many web-search-engines retrieve similar images by searching and matching textual metadata associated with digital images. For better precision of the retrieved resultant images, this type of search requires associating meaningful image-descriptive-text-labels as metadata with all images of the database [2].

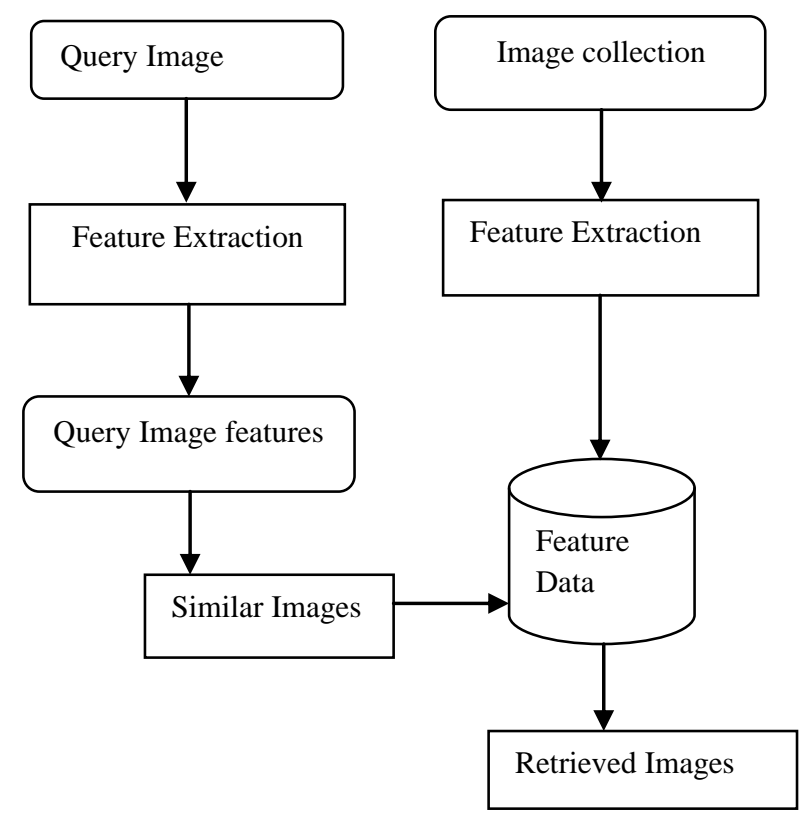

Fig.1. Architectural view of Content based image retrieval system

CBIR is originated from fields such as statistics, pattern recognition, signal processing and Image Processing. "Content-based" means that the search will analyze the actual contents of the image.

The term 'content' in this context refers to colors, shapes, textures, or any other information that can be derived from the image itself. It involves two steps:

Feature Extraction: First step is process of extracting image features to a distinguishable extent.

Matching: Second step involves matching these features to yield a result that is visually similar.

Fig 2 shows the basic CBIR model. CBIR systems search collection of images based on features that can be extracted from the image files themselves without manual descriptive. In past decades many CBIR systems have been developed, the 
common ground for them is to extract a desired image. Comparing two images and deciding if they are similar or not is a relatively easy thing to do for a human. Getting a computer to do the same thing effectively is however a different matter [3]

"Content-based" means that the search will analyze the actual contents of the image. The term 'content' in this context might refer colors, shapes, textures, or any other information that can be derived from the image itself. Without the ability to examine image content, searches must rely on metadata such as captions or keywords, which may be laborious or expensive to produce [4]

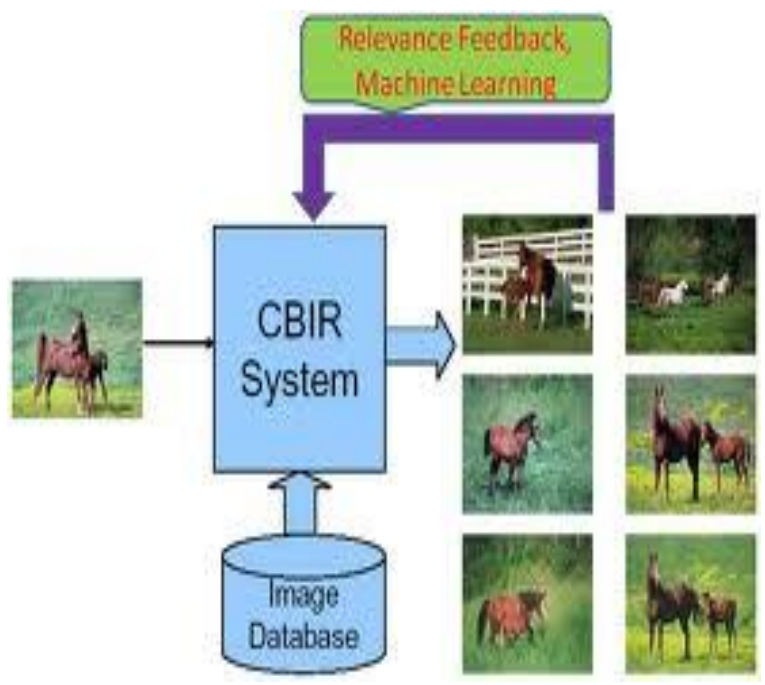

Fig.2. Basic CBIR system

\section{CONTENT COMPARISON TECHNIQUES}

The sections below describe common methods for extracting content from images so that they can be easily compared. The methods outlined are not specific to any particular application domain.

Color: Examining images based on the colors they contain is one of the most widely used techniques because it does not depend on image size or its orientation.

Texture: Texture measures look for visual patterns in images and how they are spatially defined.

Shape: Shape does not refer to the shape of an image but to the shape of a particular region that is being sought out.

Based on above parameters like color, shape and texture, a Content Based Image Retrieval System (CBIR) will be developed which will analyze both color and grayscale images, and user will be able to execute a query by example and get multiple results in ascending order of matching the various parameters.

\section{CBIR-AN OVERVIEW}

Content-based image retrieval, a technique which uses visual contents to search images from large scale image databases according to users' interests, has been an active and fast advancing research area since the 1990s.

The driving forces

- Internet

- $\quad$ Storage devices
- $\quad$ Computing power

Two approaches

- Text-based approach

- Content-based Approach

Data Scope Understanding the nature and scope of image data plays a key role in the complexity of image search system design.. Along this dimension, we classify search data into the following categories.

- Personal Collection. This consists of a largely homogeneous collection generally small in size,and usually stored on a local storage media.

- Domain-Specific Collection. This is a homogeneous collection providing access to controlled users with very specific objectives.

- $\quad$ Enterprise Collection. We define this as a heterogeneous collection of pictures accessible to users within an organization's intranet.

- Web. World Wide Web pictures are accessible to practically everyone with an Internet connection. Current WWW image search engines such as Google and Yahoo!

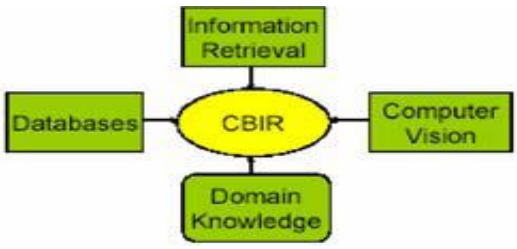

Fig.3. Content-Based Image Retrieval (CBIR) and Annotation System

\subsection{Query Modalities and Processing}

In the realm of image retrieval, an important parameter to measure user-system interaction level is the complexity of queries supported by the system.

- Keywords: This is a search in which the user poses a simple query in the form of a word or bigram.

- Free-Text: This is where the user frames a complex phrase, sentence, question, or story about what she desires from the system.

\section{PROBLEM FORMULATED}

After the studying about the different aspects of an image and the related work of other researchers in the area, the need is felt to develop a system which efficiently retrieves images based on the critical parameters of images like Color, Texture or Shape. Many of the researches already worked on the individual parameter to retrieve the image or the just few combination, it is the need to work with all the three critical parameters for more accuracy of the results in turn the system developed will be generalized. To solve the above stated problem a "Query Based Image Management System" (QBIMS) has been developed with the following combinations:

1. Retrieval based on Texture features

- $\quad$ Entropy

- $\quad$ Energy

- Contrast

2. Retrieval based on Shape features 
- Horizontal Edges

- $\quad$ Vertical Edges

3. Retrieval based on Color features

- Mean Deviation

- Standard Deviation

- Median

- Histogram

4. Retrieval based on combination of Texture and Shape features

5. Retrieval based on combination of Texture and Color features

6. Retrieval based on combination of Shape and Color features

Based on the analysis of the above mentioned researche the objectives of the work are as follows:

- $\quad$ Analyze the current CBIR system in detail

- Formulate the new concept based on Colour, Texture and Shape parameter

- Implement the new system using MATLAB as CBIR Compare and Analyze the results.

\section{METHODOLOGY}

After the studying about the different aspects of images and the related work of other researchers in this area we found that many of the researches already worked on the individual parameter to retrieve the image i.e. "Color, Shape and Texture" or the just few combination. To solve the problem a "Query Based Image Management System" (QBIMS) has been developed with the following combinations: and one combination is discuss.

\section{Retrieval Based On Texture Features}

The three features of texture which have been used in the accomplishment of the proposed solution from the problem statement of the work undertaken in this work are:

\section{Energy \\ 2. Entropy \\ 3. Contrast}

It will store the extracted values into an excel file in the form of matrix of order $n$ by 3 where $n$ is the number of images in the image database. It will then compares the extracted values of the query image as well as that of the images stored in excel file.

\section{Energy}

img_rgb = imread(path_img);

img_data $=$ img_rgb;

[xx yy zz] = size(img_data);

tp $=x x^{*} y y$;

gray_img = rgb2gray(img_data);

texture_img = rangefilt(gray_img);

texture_energy $=$ sum(texture_img(:).^2)/tp;

Here we are extracting the energy of the image for the retrieval purpose

Here, tp=total pixel

\section{Entropy}

texture entropy $=$ entropy(texture_img);

Here we are extracting the entropy of the image for the retrieval purpose.

In information theory, entropy is a measure of the uncertainty in a random variable. In this context, the term usually refers to the Shannon entropy, which quantifies the expected value of the information contained in a message. Entropy is typically measured in bits, nats, or bans. Shannon entropy is the average unpredictability in a random variable, which is equivalent to its information content. The concept was introduced by Claude E. Shannon in his 1948 paper "A Mathematical Theory of Communication". Shannon entropy provides an absolute limit on the best possible lossless encoding or compression of any communication, assuming that the communication may be represented as a sequence of independent and identically distributed random variables. Shannon's source coding theorem shows that, in the limit, the average length of the shortest possible representation to encode the messages in a given alphabet is their entropy divided by the logarithm of the number of symbols in the target alphabet [5].

Entropy is a measure of unpredictability or information content. To get an informal, intuitive understanding of the connection between these three English terms, consider the example of a poll on some political issue. Usually, such polls happen because the outcome of the poll isn't already known. In other words, the outcome of the poll is relatively unpredictable, and actually performing the poll and learning the results gives some new information; these are just different ways of saying that the entropy of the poll results is large. Now consider the case that the same poll is performed a second time shortly after the first poll. Since the result of the first poll is already known, the outcome of the second poll can be predicted well and the results should not contain any new information; in this case the entropy of the second poll results is small [6].

\section{Contrast}

cmap_img = contrast (texture_img);

Here we are extracting the contrast of the image for the retrieval purpose range filter is used to filter the gray image and contrast is the inbuilt function of the MATLAB.

Contrast is the difference in luminance and/or color that makes an object (or its representation in an image or display) distinguishable. In visual perception of the real world, contrast is determined by the difference in the color and brightness of the object and other objects within the same field of view. Because the human visual system is more sensitive to contrast than absolute luminance, we can perceive the world similarly regardless of the huge changes in illumination over the day or from place to place. The maximum contrast of an image is the contrast ratio or dynamic range [7].

\subsection{Proposed Algorithm}

Algorithm (TBIR)

1. Insert the query image.

2. Convert the image from RGB to grey scale to use it

3. Calculate the pixel of image 
4. Extract all the features of image like Image Entropy, Image Energy and Image Contrast into matrix format

5. Calculate the feature matrices' and store it into a excel sheet

6. Now compare these feature matrices with already exist feature of the database images

If (features are matched)

Show all the which are matching the feature in output GUI window

Else

Report an error that match not found

7. Go to step 2 for every new process.

8. End of process

\section{IMPLEMENTATION}

The performance analysis of all the seven modules is being done on the basis of two criteria's:

\section{Precisio \\ 2. Recall}

Precision $=$ number of relevant images retrieved $/$ total number of images.

Recall $=$ number of relevant images retrieved /total number of relevant images.

This performance is being shown in both the tabular as well graphical form below:

Total number of images $=100$

Number of relevant images $=10$

Table. I. Precision and Recall

\begin{tabular}{|l|l|l|}
\hline \multicolumn{1}{|c|}{ TECHNIQUES } & PRECISION & RECALL \\
\hline $\begin{array}{l}\text { Texture Based } \\
\text { ImageRetrieval }\end{array}$ & $\mathbf{0 . 0 6}$ & $\mathbf{0 . 6 0}$ \\
\hline $\begin{array}{l}\text { ShapeBased Image } \\
\text { Retrieval }\end{array}$ & $\mathbf{0 . 0 4}$ & $\mathbf{0 . 4 0}$ \\
\hline $\begin{array}{l}\text { Color Based } \\
\text { ImageRetrieval }\end{array}$ & $\mathbf{0 . 0 6 5}$ & $\mathbf{0 . 6 5}$ \\
\hline $\begin{array}{l}\text { Texture and ShapeBased } \\
\text { ImageRetrieval }\end{array}$ & $\mathbf{0 . 0 8 6}$ & $\mathbf{0 . 8 6}$ \\
\hline $\begin{array}{l}\text { Shape and Color Based } \\
\text { Image Retrieval }\end{array}$ & $\mathbf{0 . 0 7 2}$ & $\mathbf{0 . 7 2}$ \\
\hline $\begin{array}{l}\text { Texture and ColorBased } \\
\text { ImageRetrieval }\end{array}$ & $\mathbf{0 . 0 7}$ & $\mathbf{0 . 7 0}$ \\
\hline
\end{tabular}

Graphical Form

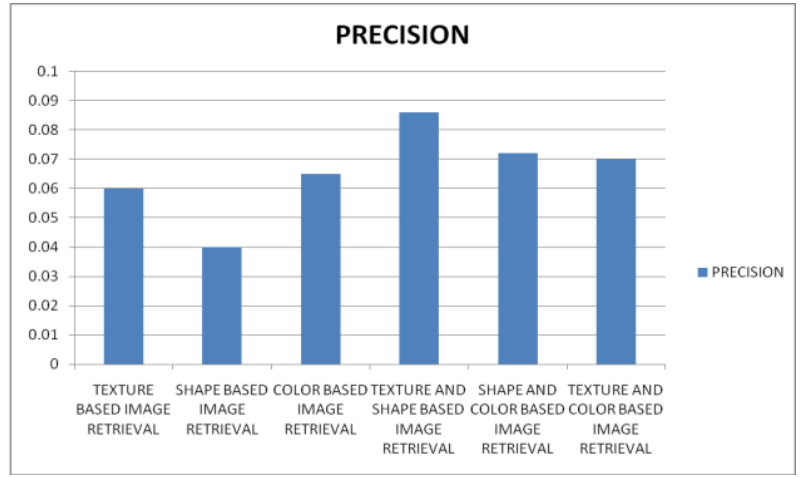

Graph .1. Comparative graph of Precision in all Cases

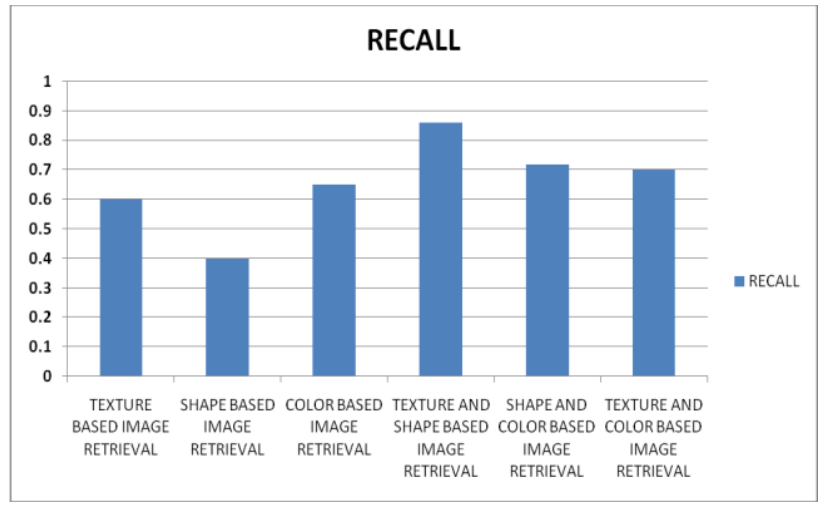

Graph.2. Comparative graph of Recall in all Cases

\section{Result Analysis}

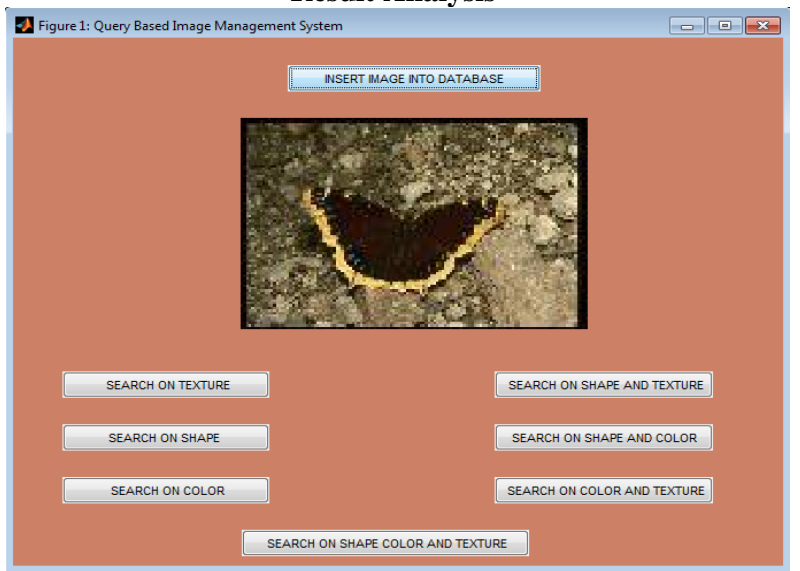

Fig.4. inserting an Image GUI

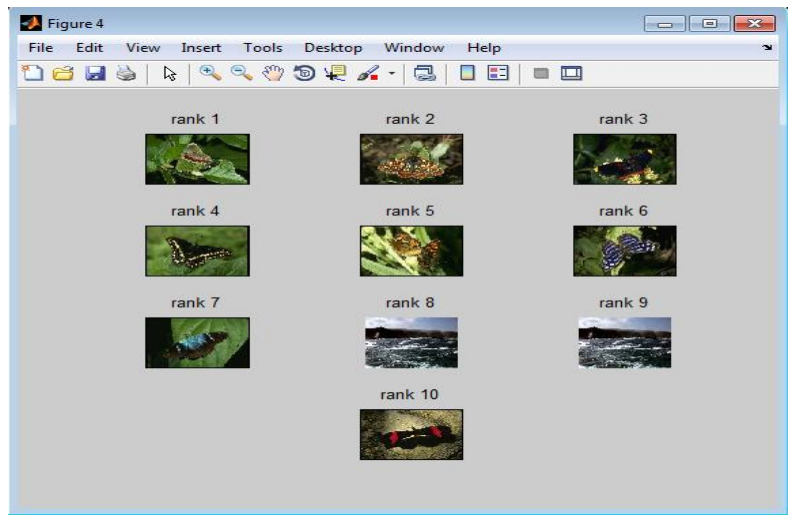

fig5. Texture Based Image Retrieval Output 


\section{CONCLUSION}

Content based image retrieval is one of the most focused and growing research areas of the content based Image Processing. The CBIR tools can be utilized in numerous applications such as digital libraries, photo sharing sites and crime prevention. In this paper, an honest attempt is made to develop, implement and integration of various novel algorithms to result into Graphical User Interface based tool for processing of select a single query image for retrieval of similar images has been achieved successfully. High performance measures Precision and Recall, computed with respect to Ground Truth foreground for extracted foreground with proposed method endorses the uniqueness and effectiveness of the method \& produced results. This tool is very effective in selecting a very similar image from image database.

\section{REFERENCES}

[1] Gaurav Jaswal Asmit Kaul , “ Content Based Image Retrieval ", National Conference on Computing, Communication and Control, A Literature Review, National Institute of Technology, Hamirpur- 177001, Himachal Pradesh(India).

[2] R.Senthil Kumar, Dr.M.Senthilmurugan, "Content-Based Image Retrieval System in Medical",International Journal of Engineering Research \& Technology (IJERT),Vol. 2 Issue 3, March - 2013,ISSN: 2278-0181.

[3] Ivan Lee, Paisarn Muneesawang, Ling Guan, "Automatic Relevance Feedback for Distributed Content-Based Image Retrieval",ICGST, ieee.org FLEXChip Signal Processor (MC68175/D), Motorola, 1996.
[4] Paolo Parisen Toldin, "A survey on contentbased image retrieval/browsing systems exploiting semantic", 201009-13.

[5] M. Sifuzzaman, M.R. Islam and M.Z. Ali ,"Application of Wavelet Transform and its Advantages Compared to Fourier Transform ", Journal of Physical Sciences, Vol. 13, 2009, 121-134 ISSN: 0972- 8791 .

[6] Pooja Verma, Manish Mahajan, "Retrieval of better results by using shape techniques for content based retrieval”,IJCSC ,Vol. 3, No.2, January-June 2012, pp. 254-257, ISSN: 0973-7391. [14] [14] Nidhi Singhai,Prof. Shishir K. Shandilya , "A Survey On: Content Based Image Retrieval Systems ", International Journal of Computer Applications (0975 - 8887) Volume 4 - No.2, July 2010.

[7] Jean-Francois Omhover, Marcin Detyniecki,University P. et M. Curie - CNRS, rue du Capitaine Scott, "Combining text and image retrieval".

[8] Ryszard S. Chora's, "Image Feature Extraction Techniques and Their Applications for CBIR and Biometrics Systems", International Journal of Biology and Biomedical Engineering Issue 1, Vol. 1, 2007.

[9] Swapnalini Pattanaik, Prof.D.G.Bhalke, "Beginners to Content Based Image Retrieval", International Journal of Scientific Research Engineering \&Technology (IJSRET),Volume 1 Issue2 pp 040-044 May 2012 www. ijsret.org ISSN 2278 - 0882,IJSRET ,2012. 